\title{
UNBS5162 inhibits the proliferation of esophageal cancer squamous cells via the PI3K/AKT signaling pathway
}

\author{
$\mathrm{DAN} \mathrm{HE}^{1}$ and SUOLIN $\mathrm{ZHANG}^{2}$ \\ ${ }^{1}$ Department of Thoracic Surgery, Affiliated Tumor Hospital, Xinjiang Medical University, Urumqi, Xinjiang 830011; \\ ${ }^{2}$ Department of Thoracic Surgery, The Second Hospital of Shandong University, Jinan, Shandong 250033, P.R. China
}

Received June 29, 2017; Accepted September 14, 2017

DOI: $10.3892 / \mathrm{mmr} .2017 .7893$

\begin{abstract}
C-X-C motif chemokine ligand (CXCL) signaling has been demonstrated to be involved in cancer invasion and migration; therefore, CXCL antagonists may serve as anticancer drugs by preventing tumor proliferation. The present study aimed to investigate whether a pan antagonist of CXCLs, UNBS5162, may inhibit esophageal cancer proliferation and to identify the underlying mechanisms. Cell proliferation and cell colony formation results, which were determined by a Cell Counting Kit-8 assay and crystal violet staining, respectively, demonstrated that UNBS5162 inhibited esophageal cancer cell proliferation. Following treatment with UNBS5162, Transwell migration and Matrigel invasion assays, and flow cytometry with Annexin V-fluorescein isothiocyanate and propidium iodide staining, were performed to investigate cell migration, invasion and apoptosis in human esophageal cancer cells. The results indicated that invasion and migration was reduced in UNBS5162-treated cells, while apoptosis was increased. Western blotting experiments confirmed that UNBS5162 downregulated the protein expression of proteins associated with the phosphatidylinositol 3-kinase (PI3K)/AKT signaling pathway, including the levels of phosphorylated (p)-AKT, p-mechanistic target of rapamycin kinase, ribosomal protein S6 kinase $\beta 1$ and cyclin D1. In addition, upregulated expression of programed cell death 4 was observed following UNBS5162 treatment. The present study demonstrated that UNBS5162 is a novel naphthalimide that may have potential therapeutic use for the prevention of esophageal cancer proliferation and metastasis via the PI3K/AKT signaling pathway.
\end{abstract}

Correspondence to: Professor Suolin Zhang, Department of Thoracic Surgery, The Second Hospital of Shandong University, 247 Beiyuan Road, Jinan, Shandong 250033, P.R. China

E-mail: suolinz@163.com

Key words: UNBS5162, chemokine, naphthalimide, esophageal cancer, proliferation, invasion, migration, apoptosis, phosphatidylinositol 3-kinase/AKT

\section{Introduction}

Combined, digestive tract tumors are currently the most common group of tumor types, and the incidence and mortality accounts for $\sim 30 \%$ of all tumors worldwide (1). Esophageal cancer, as one of the most common digestive tract malignancies, is the 6th leading cause of cancer-associated mortality globally (2). Two pathological types of esophageal cancer exist, which are esophageal adenocarcinoma and esophageal squamous cell carcinoma. Geographical distribution differences in the incidence of esophageal cancer are observed; in western countries, the incidence of esophageal adenocarcinoma is predominant, while in East Asia the incidence of esophageal squamous cell carcinoma predominates (3). Despite advanced treatment with concurrent chemotherapy alone or as an adjunct to radiotherapy or surgery, the 5-year survival rate is $<20 \%$ for patients with esophageal cancer (4). Thus, the development of chemotherapy drugs with enhanced efficacies is urgently required for esophageal cancer.

Chemokines consist of a family of $\sim 50$ low-molecular-weight chemotactic cytokines (5). The chemokine family is divided into four separate subtypes, including C-X-C (CXC), C-C (CC), $\mathrm{C}-\mathrm{X} 3-\mathrm{C}(\mathrm{CX} 3 \mathrm{C})$ and $\mathrm{X}-\mathrm{C}(\mathrm{XC})$ motif chemokine ligands, where $\mathrm{X}$ represents any amino acid positioning of the conserved cysteines in the aminoterminal region of these proteins (6). At present, the CXC motif chemokine ligands (CXCLs) that have been identified are termed CXCL1-CXCL17 $(7,8)$. Chemokines have indirect roles in tumor development by affecting angiogenesis and tumor-leukocyte interactions, and also have direct effects on tumor transformation, survival and growth, and invasion and metastasis. Various studies have reported that CXCLs are overexpressed in numerous tumor types, including esophageal cancer. Bruyère et al (9) revealed that the proangiogenic CXCL1, CXCL2, CXCL8, CXCL16 and CXC motif receptor (CXCR) 4 were markedly overexpressed in esophageal cancer cells. Furthermore, CXCL12 and its receptor, CXCR4, are reported to be implicated in the metastasis of esophageal squamous cell carcinoma to lymph nodes (10). Notably, Wang et al (11) reported that CXCL1-CXCR2 and CXCL2-CXCR2 signaling is associated with increased esophageal cancer cell proliferation and may therefore be implicated in the development of esophageal tumors. Chemokine antagonists prevent the binding of chemokines and chemokine receptors, and thus have therapeutic effects in various tumor 
types. Based on these previous observations, the present study aimed to identify an efficient chemokine antagonist as a potential effective chemotherapy drug for human esophageal cancer.

UNBS5162 is a pan antagonist of CXCLs and is also a naphthalimide. As an antagonist of CXCL, to the best of our knowledge, the effect of UNBS5162 in esophageal squamous cell carcinoma has not previously been reported. Furthermore, as a naphthalimide, the effect of UNBS5162 in other tumor types is scarcely reported, including esophageal cancer. Therefore, the present study aimed to investigate the effect of UNBS5162 on the proliferation and metastasis of esophageal squamous cell carcinoma, in addition to the underlying mechanisms of UNBS5162-induced antitumor effects.

\section{Materials and methods}

Cell culture. The KYSE-30 human esophageal squamous cell carcinoma cell line was purchased from the Cell Bank of the Type Culture Collection of the Chinese Academy of Sciences (Shanghai, China). Cells were cultured with Dulbecco's modified Eagle's medium (DMEM; Gibco; Thermo Fisher Scientific, Inc., Waltham, MA, USA) at $37^{\circ} \mathrm{C}$ with an atmosphere of $5 \% \mathrm{CO}_{2}$ in a humidified cell incubator. The medium contained $10 \%$ fetal bovine serum (FBS; Gibco; Thermo Fisher Scientific, Inc.), $100 \mathrm{U} / \mathrm{ml}$ penicillin and $0.1 \mathrm{mg} / \mathrm{ml}$ streptomycin (both from Sigma-Aldrich; Merck KGaA, Darmstadt, Germany). When the cells reached the logarithmic growth phase, cells were washed three times with PBS and subsequently digested with $0.25 \%$ trypsin (Beijing Solarbio Science and Technology Co., Ltd., Beijing, China). When the cells became round, culture medium was added to terminate the digestion. Cells were subsequently resuspended to a single cell suspension and seeded in 6-well plates for subsequent experiments. When the cell density was $\sim 80 \%, 10 \mu \mathrm{M}$ UNBS5162 (Medchem Express, South Brunswick, NJ, USA) was added to cells. The negative control (NC) group was treated with dimethyl sulfoxide (DMSO; 1:1,000; Amresco, Solon, OH, USA) instead.

Cell proliferation assay. Cell suspension $(100 \mu \mathrm{l})$ was routinely seeded into 96 -well plates $\left(1 \times 10^{3}\right.$ cells/well). Cells were treated with $10 \mu \mathrm{M}$ UNBS5162 or DMSO $(1: 1,000)$ which were incubated at $37^{\circ} \mathrm{C}$ with an atmosphere of $5 \% \mathrm{CO}_{2}$ in a humidified cell incubator. Cell viability was detected every $24 \mathrm{~h}$ for a total of $72 \mathrm{~h}$. Prior to detection, $10 \mu \mathrm{l}$ Cell Counting Kit-8 (CCK-8) reagent (Beijing Solarbio Science and Technology Co., Ltd.) was added to cells and incubated at $37^{\circ} \mathrm{C}$ for $90 \mathrm{~min}$, subsequently, the optical density value at $450 \mathrm{~nm}$ was detected and a proliferation curve was plotted.

Cell colony formation. Cell suspensions containing 500 cells were cultured in $60 \mathrm{~mm}$ dishes containing $5 \mathrm{ml}$ prewarmed culture medium at $37^{\circ} \mathrm{C}$. Dishes were gently rotated to disperse the cells homogeneously. Cells were treated with $10 \mu \mathrm{M}$ UNBS5162 for $48 \mathrm{~h}$ at $37^{\circ} \mathrm{C}$ in an atmosphere of $5 \% \mathrm{CO}_{2}$ and $95 \%$ air. Following treatment, media was removed and cells were washed twice with PBS. Then cells were maintained in DMEM with $10 \%$ FBS for $1-2$ weeks at $37^{\circ} \mathrm{C}$ in a humidified cell incubator with an atmosphere of $5 \% \mathrm{CO}_{2}$. When visible clones were observed, culture was terminated, the supernatant was discarded and cells were washed twice with PBS. Cells were subsequently fixed with $4 \%$ paraformaldehyde for $30 \mathrm{~min}$ at room temperature, followed by gentle washing with PBS three times and staining with $0.1 \%$ crystal violet for $30 \mathrm{~min}$ at room temperature. Cells were washed with running water and processed by air drying. Visible colonies consisting of $\geq 50$ cells were counted and the size and number of clones were compared.

Invasion and migration assays. Cancer cell invasion assays were performed using 24-well Transwell plates. Matrigel matrix gel (100 $\mu \mathrm{l}$; BD Biosciences, Franklin Lakes, NJ, USA) was diluted with serum-free DMEM overnight at 1:6 and was added to the upper chamber of the 24-well plate in the Transwell chamber. Following treatment of cells with $10 \mu \mathrm{M}$ UNBS5162 for $48 \mathrm{~h}$ at $37^{\circ} \mathrm{C}$ with an atmosphere of $5 \% \mathrm{CO}_{2}$ in a humidified cell incubator, $1 \times 10^{5}$ cells were seeded into the upper chambers resuspended in $100 \mu \mathrm{l}$ serum-free DMEM. DMEM $(500 \mu \mathrm{l})$ with $10 \%$ FBS was added to lower chambers. Following incubation at $37^{\circ} \mathrm{C}$ overnight, non-invading cells in the upper chambers were removed by scrubbing gently using a cotton-tipped swab. The cells that invaded the lower chambers were fixed in $4 \%$ paraformaldehyde for $30 \mathrm{~min}$ at room temperature and stained with $0.1 \%$ crystal violet for $20 \mathrm{~min}$ at room temperature. Following washing with PBS, the number of invading cells was determined by light microscope in five random fields for each insert (magnification, x100) and calculated as the mean number of cells per field.

The protocol for the migration assays was similar to the invasion assay, however, Matrigel matrix was not added to the upper chamber of Transwell inserts and the number of cells seeded in the upper chambers was $5 \times 10^{3}$.

Flow cytometry analysis. Following treatment of cells with $10 \mu \mathrm{M}$ UNBS5162 for $48 \mathrm{~h}$ at $37^{\circ} \mathrm{C}$, the medium was removed and replaced with serum-free DMEM. The cells were starved for $24 \mathrm{~h}$ under normal conditions. Cells were digested by trypsin without EDTA, collected into a centrifuge tube and centrifuged at $800 \mathrm{x}$ g for $5 \mathrm{~min}$ at $4^{\circ} \mathrm{C}$. Cells were resuspended in PBS that was precooled at $4^{\circ} \mathrm{C}$, centrifuged at $800 \mathrm{x}$ g at $4^{\circ} \mathrm{C}$ for 5 min again and the supernatant was carefully aspirated. Subsequently, $1 \mathrm{X}$ binding buffer diluted by $4 \mathrm{X}$ binding buffer [Annexin V-fluorescein isothiocyanate (FITC)/propidium iodide (PI) kit; both from Beijing 4A Biotech, Co., Ltd., Beijing, China] was used to resuspend the cells at a density of $1-5 \times 10^{6}$ cells $/ \mathrm{ml}$ at $4^{\circ} \mathrm{C}$. Cell suspension $(100 \mu \mathrm{l})$ and Annexin V-FITC (5 $\mu \mathrm{l}$; Annexin V-FITC/PI kit; Beijing 4A Biotech, Co., Ltd.) were added to a $5 \mathrm{ml}$ flow tube and, following mixing, incubated at room temperature in the dark for $5 \mathrm{~min}$. Subsequently, $10 \mu \mathrm{l}$ PI (Annexin V-FITC/PI kit; Beijing 4A Biotech, Co., Ltd.) was added for incubation at room temperature in the dark for 5 min and subsequently, $400 \mu \mathrm{l}$ PBS was added for detection. Results were analyzed and processed with FlowJo software (version 7.6.3; FlowJo, LLC, Ashland, OR, USA).

Western blot analysis. After the $\mathrm{NC}$ and the experimental (10 $\mu \mathrm{M}$ UNBS5162) groups were treated for $48 \mathrm{~h}, 6$-well plates were placed on ice and treated with radioimmunoprecipitation assay (RIPA) lysis buffer (CWBiotech Co., Ltd., Beijing, China) with the addition of protease inhibitors (Protease Inhibitor Cocktail; CWBiotech Co., Ltd.). Subsequently, the 
A

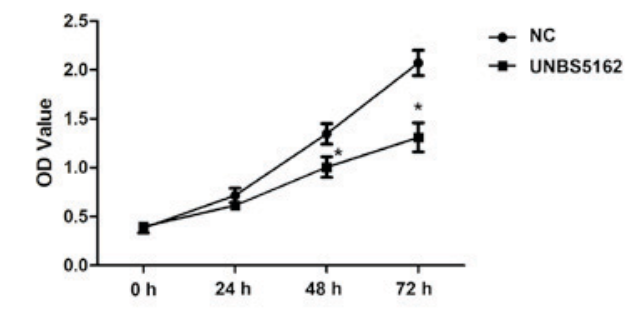

B

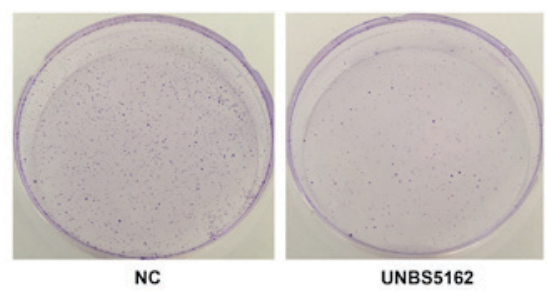

C

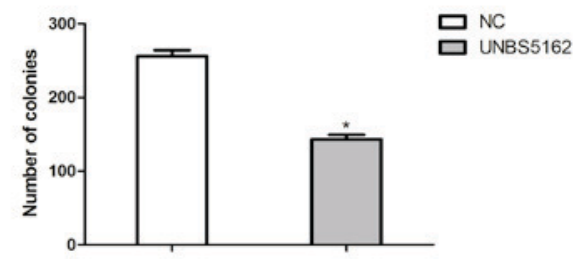

Figure 1. UNBS5162 inhibits squamous cell esophageal cancer growth (A) KYSE-30 squamous cell esophageal cancer cell proliferation was investigated using a Cell Counting Kit-8 assay. (B) Representative images of stained colonies in colony formation assay. (C) Number of KYSE-30 colonies counted in cell colony formation assay. Data are presented as the mean \pm standard deviation. " $\mathrm{P}<0.05$ vs. NC group. NC, negative control; OD, optical density.

concentration of extracted protein was measured by a BCA kit. Extracted proteins $(20 \mu \mathrm{g})$ were mixed with loading buffer (5X; Thermo Fisher Scientific, Inc.) and boiled at $95^{\circ} \mathrm{C}$ for $5 \mathrm{~min}$. The mixture of denatured protein was separated by $10-20 \%$ polyacrylamide gels (Bio-Rad Laboratories, Inc., Hercules, CA, USA) and transferred to a polyvinylidene fluoride membrane (PVDF; Thermo Fisher Scientific, Inc.). Membranes were blocked with 5\% non-fat milk for $1 \mathrm{~h}$ at room temperature and incubated with primary antibodies against Bcl-2 (1:1,000, cat. no. 12789-1-AP), Bcl-2-associated X (Bax; 1:1,000, cat. no. 23931-1-AP), and cleaved-caspase-3 (1:1,000, cat. no. 25546-1-AP) (all from ProteinTech Group, Inc., Chicago, IL, USA), AKT (1:1,000, cat. no. 4691), phosphorylated (p)-AKT (1:1,000, cat. no. 4060), mechanistic target of rapamycin kinase (mTOR; 1:1,000, cat. no. 2983), and p-mTOR (1:1,000, cat. no. 5536) (all from Cell Signaling Technology, Inc., Danvers, MA, USA), ribosomal protein S6 kinase $\beta 1$ (p70 ${ }^{\mathrm{S} 6 \mathrm{~K}}, 1: 1,000$, cat. no. 14485-1-AP), programed cell death 4 (PDCD4; 1:3,000, cat. no. 12587-1-AP), cyclin D1 (1:5,000, cat. no. 60186-1-Ig) and GAPDH (1:5,000, cat. no. 10494-1-AP) (all from ProteinTech Group, Inc.) overnight at $4^{\circ} \mathrm{C}$. Membranes were washed with PBS with Tween-20 (PBST) three times for $5 \mathrm{~min}$ and incubated with polyclonal horseradish peroxidase-conjugated goat anti-rabbit $(1: 10,000$, cat. no. SA00001-2) and goat anti-mouse (1:10,000, cat. no. SA00001-1) (both from ProteinTech Group, Inc.) secondary antibodies for $1 \mathrm{~h}$ at room temperature. Following a final

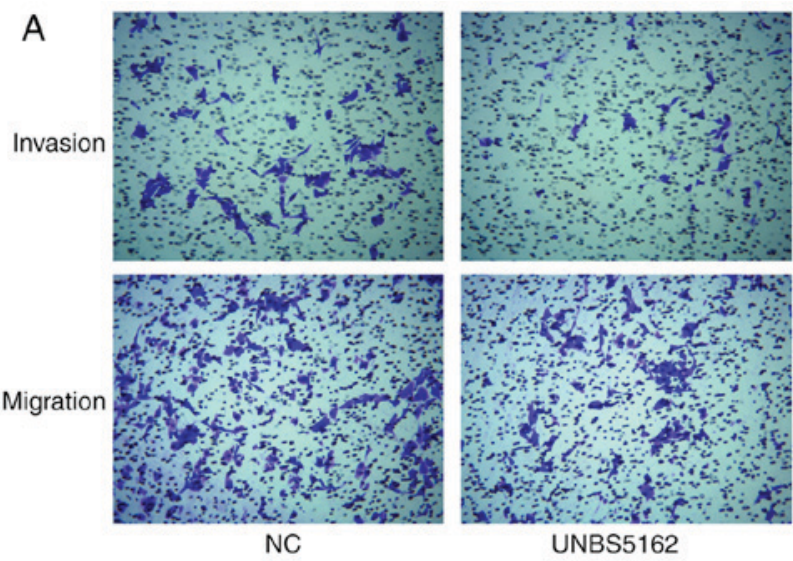

B

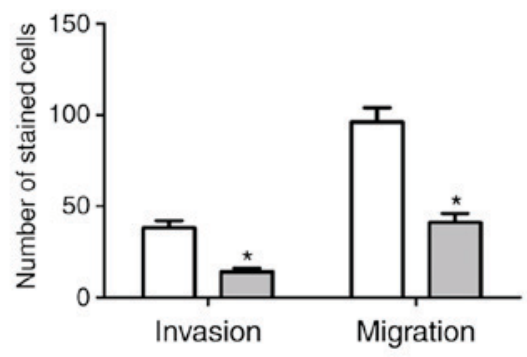

Figure 2. UNBS5162 inhibits KYSE-30 squamous cell esophageal cancer invasion and migration. (A) Representative images of lower chambers following invasion and migration assays were captured using an inverted light microscope at x100 magnification. (B) Number of stained cells in lower chambers following invasion and migration assays. Data are presented as the mean \pm standard deviation, $\mathrm{n}=3 ;{ }^{*} \mathrm{P}<0.05$ vs. $\mathrm{NC}$ group. $\mathrm{NC}$, negative control.

washing step, signals were developed by enhanced chemiluminescence reagent (PerkinElmer, Inc., Waltham, MA, USA) and quantified using Quantity One software (version 4.62; Bio-Rad Laboratories, Inc.), with GAPDH as an internal control.

Statistical analysis. SPSS 18.0 statistical analysis software (SPSS, Inc., Chicago, IL, USA) was used to analyze the experimental data. Data are presented as the mean \pm standard deviation. Comparisons between the two groups were performed using Student's t-tests. $\mathrm{P}<0.05$ was considered to indicate a statistically significant difference.

\section{Results}

UNBS5162 inhibits the proliferation and colony formation of KYSE-30 squamous cell esophageal carcinoma cells. To investigate the effect of UNBS5162 on cell growth, the proliferation of KYSE-30 cells treated with $10 \mu \mathrm{M}$ UNBS5162 was investigated using a CCK-8 assay. As demonstrated in Fig. 1A, the proliferation of UNBS5162-treated KYSE-30 cells was significantly inhibited at 48 and $72 \mathrm{~h}$, compared with the NC group $(\mathrm{P}<0.05)$. Furthermore, the inhibitory effects of UNBS5162 on the cell proliferation occurred in a time-dependent manner (Fig. 1A). To further investigate whether UNBS5162 has an effect on the survival of adherent cells and the number of formation of clones, a cell colony formation assay was performed. The results of the colony formation assay demonstrated that the ability of KYSE-30 cells to form colonies was significantly inhibited in the UNBS5162 group, compared with the $\mathrm{NC}$ group $(\mathrm{P}<0.05$; Fig. $1 \mathrm{~B}$ and $\mathrm{C})$. The results presented 
A
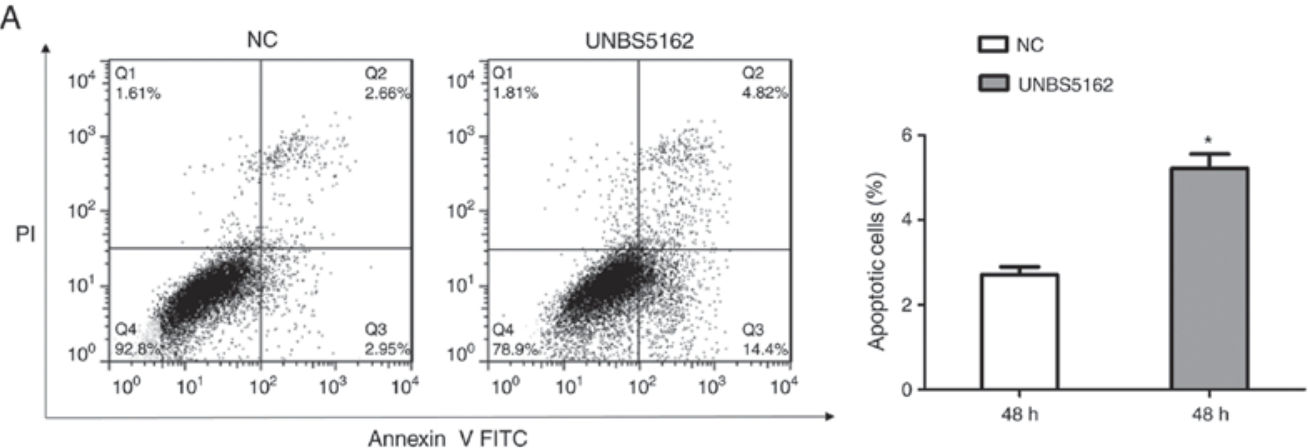

B

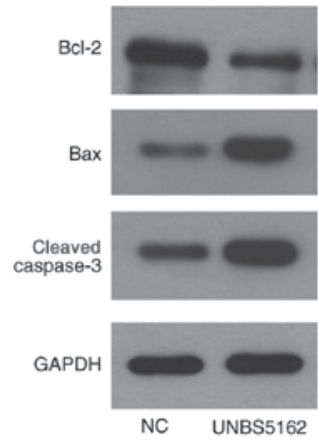

C

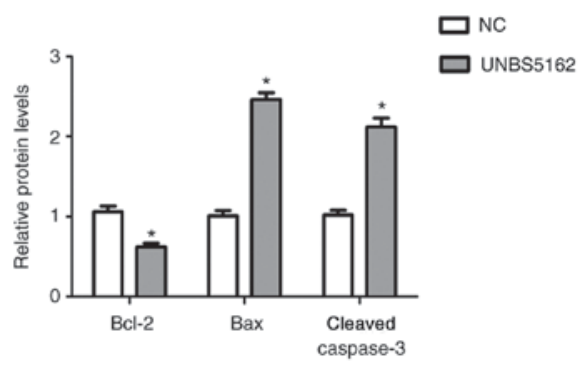

Figure 3. UNBS5162 induces KYSE-30 squamous cell esophageal carcinoma apoptosis. (A) KYSE-30 cells were treated with UNBS5162 and labeled with Annexin V/FITC and PI, which was followed by flow cytometric analysis to quantify the percentage of apoptotic cells in each group. The numbers in quadrants of Q2 and Q4 were considered to indicate apoptotic cells. (B) Representative western blot images for Bcl-2, Bax, and cleaved caspase-3 protein expression in KYSE-30 cells treated with $10 \mu \mathrm{M}$ UNBS5162 for $24 \mathrm{~h}$. (C) Protein band intensities were quantified and the results were normalized to the GAPDH loading control. Protein expression is presented relative to the $\mathrm{NC}$ group. ${ }^{*} \mathrm{P}<0.05$ vs. NC. Data are presented as the mean \pm standard deviation, $\mathrm{n}=3$. FITC, fluorescein isothiocyanate; PI, propidium iodide; Bax, Bcl-2-associated X; NC, negative control.

in Fig. 1 indicated that UNBS5162 was able to suppress the cell proliferation and the ability of squamous cell esophageal carcinoma cells to form colonies.

UNBS5162 inhibits the migration and invasion of KYSE-30 squamous cell esophageal carcinoma cells. As UNBS5162 inhibited the proliferation and colony formation of KYSE-30 cells, the effects of UNBS5162 on KYSE-30 cell migration and invasion were subsequently investigated. The results of the Matrigel invasion assay demonstrated that the invasion ability of KYSE-30 cells treated with UNBS5162 was significantly inhibited compared with the $\mathrm{NC}$ group $(\mathrm{P}<0.05$; Fig. 2). In addition, the migration ability of KYSE-30 cells treated with UNBS5162 was also significantly inhibited compared with the NC group $(\mathrm{P}<0.05$; Fig. 2). These results indicate that UNBS5162 significantly reduced the cell migration and invasion of KYSE-30 squamous cell esophageal carcinoma cells.

UNBS5162 promotes the apoptosis of KYSE-30 squamous cell esophageal carcinoma cells. Staining of KYSE-30 cells with Annexin V-FITC and PI, followed by flow cytometry, was performed to determine the proportion of apoptotic cells at $48 \mathrm{~h}$ after exposure to UNBS5162. Flow cytometry analysis demonstrated that the proportion of apoptotic cells were increased following treatment with UNBS5162 compared with the NC group (Fig. 3A). These results indicate that UNBS5162 may induce cell apoptosis. Various proteins participate in the apoptotic process. Therefore, to further confirm that
UNBS5162 induces apoptosis in KYSE-30 cells, the protein expression of the proapoptotic proteins cleaved-caspase-3 and $\mathrm{Bax}$, and the antiapoptotic protein $\mathrm{Bcl}-2$, was detected in KYSE-30 cells by western blotting. The results demonstrated that the expression of Bax and cleaved-caspase- 3 was increased, whereas the levels of Bcl-2 were decreased, in KYSE-30 cells treated with UNBS5162, compared with the NC group $(\mathrm{P}<0.05$; Fig. 3B and $\mathrm{C})$. Overall, these results indicate that UNBS5162 induced apoptosis in KYSE-30 squamous cell esophageal carcinoma cells.

UNBS5162 inhibits the phosphatidylinositol 3-kinase (PI3K)/AKT signaling pathway in KYSE-30 squamous cell esophageal carcinoma cells. Previous studies have demonstrated that the activation of the PI3K/AKT signaling pathway may promote apoptosis. To determine the specific signaling pathway involved in UNBS5162-mediated apoptosis in KYSE-30 cells, the present study measured the activation of key signaling proteins, including AKT, mTOR, p70 ${ }^{\mathrm{s} 6 \mathrm{~K}}$, PDCD4 and cyclin D1 by western blotting. The results demonstrated that treatment with UNBS5162 exerted limited effects on AKT and mTOR expression, however, significant decreases in p-AKT, p-mTOR, $\mathrm{p} 70^{\mathrm{S} 6 \mathrm{~K}}$ and cyclin D1 expression, and an increase in PDCD4 expression, were observed in the UNBS5162 group, compared with the NC group $(\mathrm{P}<0.05$; Fig. 4). Therefore, UNBS5162 may inhibit the PI3K/AKT signaling pathway in KYSE-30 squamous cell esophageal carcinoma cells. 
A

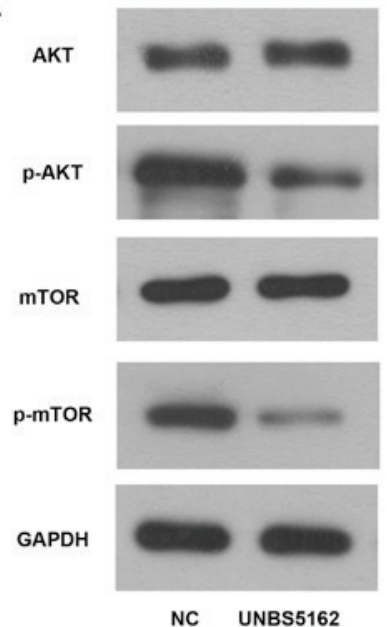

B

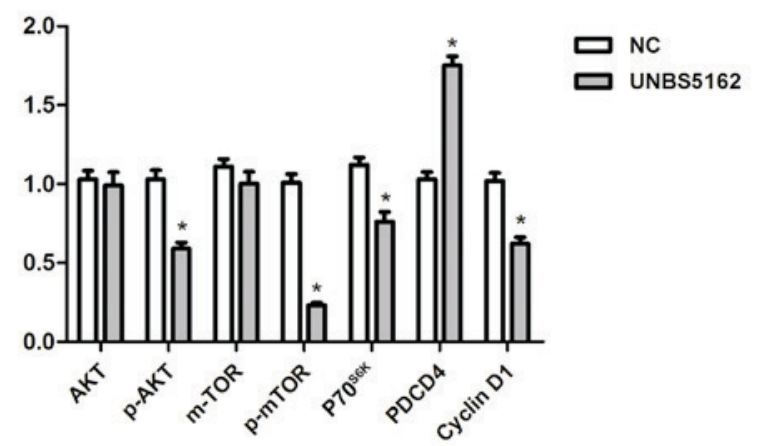

Figure 4. Effects of UNBS5162 on the PI3K/AKT signaling pathway in KYSE-30 cells. (A) Representative western blot images for AKT, p-AKT, mTOR, p-mTOR, p70 $0^{\mathrm{s} 6 \mathrm{~K}}$, PDCD4 and cyclin D1 protein expression in KYSE-30 cells treated with $10 \mu \mathrm{M}$ UNBS5162 for $24 \mathrm{~h}$. (B) Protein band intensities were quantified and the results were normalized to the GAPDH loading control. Protein expression is presented relative to the NC group. ${ }^{*} \mathrm{P}<0.05$ vs. NC. Data are presented as the mean \pm standard deviation, $\mathrm{n}=3$. PI3K, phosphatidylinositol 3-kinase; p, phosphorylated; mTOR, mechanistic target of rapamycin kinase; $\mathrm{p} 70^{\mathrm{S} 6 \mathrm{~K}}$, ribosomal protein S6 kinase $\beta 1$; PDCD4, programed cell death 4; NC, negative control.

\section{Discussion}

The results of the present study demonstrated that UNBS5162 suppressed the cell proliferation, invasion and migration of KYSE-30 esophageal cancer cells. In addition, the present study demonstrated that the antitumor effects of UNBS5162 may occur via the induction of apoptosis in KYSE-30, as protein levels of Bax and cleaved-caspase- 3 were increased, while Bcl-2 levels were decreased. Furthermore, western blotting results indicated that UNBS5162-induced apoptosis in KYSE-30 cells may occur via inhibition of the PI3K/AKT signaling pathway.

UNBS5162 is an antagonist of CXCL chemokines. Chemokines are divided into four subtypes, which include CXCL, CCL, CX3CL and XCLs. Based on the different ligands that bind to them, chemokine receptors are divided into the following four categories: CXCR, CCR, CX3CR and $\mathrm{XCR}$. These groups are based on the arrangement of cysteine residues involved in the formation of disulfide bonds (12). In the $\mathrm{CXC}$ and $\mathrm{CX} 3 \mathrm{C}$ chemokine families, 1 or 3 amino acid residues exist between the first two cysteine residues, respectively, and the CXC family includes CXCL1-17. In the XC subtype of chemokines, the first and third cysteine residues are not present and they only contain one disulfide bond. In the CC type of chemokines, the first two cysteines are juxtaposed with no amino acid residues. A novel chemokine subfamily that lacks one of the four cysteine residues that are highly conserved among chemokines was identified in the zebrafish genome and was termed the CX family (13). At present, a total of 48 chemokines are reported to exist. Chemokines are a class of small molecule proteins that consist of $\sim 70$ amino acid residues with a molecular weight of 7-12 $\mathrm{kDa}(14,15)$. Chemokine receptors are proteins that bind to chemokines. In total, $\sim 25$ human chemokine receptor genes have been identified. The number of different types of chemokines exceeds the number of receptors and certain chemokines are able to bind to multiple chemokine receptors. These receptors are expressed in various cell types and contain seven-transmembrane regional structures; they belong to the G-protein-coupled receptor superfamily and have a molecular weight of $\sim 40 \mathrm{kDa}$ (14). Chemokines exert various biological functions through binding to their respective chemokine receptors.

Chemokines were originally identified as chemotactic cytokines and were primarily thought to function in the migration of leukocytes during inflammation and immune surveillance (16). However, it has since been established that chemokines also exhibit various additional functions during physiological conditions, including roles in homeostasis, development, tissue repair and angiogenesis, and also during pathological conditions, including roles in autoimmune and inflammatory diseases (17). In addition, increasing evidence indicates that chemokines and their receptors have a strong influence on tumor development (18). Jin et al (19) reported that knockdown of CXCR6 in gastric cancer cells inhibited the cell proliferation, migration and invasion, and also reversed epithelial-mesenchymal transition (EMT). In addition, Xiang et al (20) demonstrated that cross-activation of CXCR4 and CXCR2 contributed to the migration and invasion, and EMT, of gastric cancer cells. Furthermore, CXCL5 was reported to promote osteosarcoma cell migration and invasion in autocrine- and paracrine-dependent manners (21). The use of chemokine receptor antagonists with natural ligands to chemokine receptors has received increased attention in research. In particular, the promotion of tumor cell proliferation and inhibition of tumor cell apoptosis by chemokines has been considered. For example, inhibition of CCL3 in vitro using $\alpha$-CCL3 or evasin-1 (a CCL3-binding protein) impaired tumor cell invasion (22). Furthermore, targeted disruption of CXCL13 or its receptor, CXCR5, reduced the migration and tumorigenesis of prostate cancer cells (23). Consistently, the present study employed a CXCL antagonist, UNBS5162, which was demonstrated to inhibit squamous cell esophageal carcinoma cell proliferation, invasion and migration.

In addition to being an antagonist of CXCL, UNBS5162 is also a novel naphthalimide. Naphthalimides, which are DNA intercalators, have been investigated extensively as antitumor agents (24). Among antitumor agents, naphthalimide analog derivatives are among the most promising classes of anticancer drug candidates (25-27). Amonafide, elinafide and mitonafide have reached the clinical trials stage for the treatment of various tumors and exhibited excellent antitumor activity, 
however, the majority were abandoned due to severe adverse effects such as dose-limiting or bone marrow toxicity (28). As a novel naphthalimide, UNBS5162 inhibition of tumor progression has only been reported once for prostate cancer; in vivo, UNBS5162 significantly decreased cell survival in PC-3 human prostate cancer cells and exhibited synergistic therapeutic effects when combined with paclitaxel (29). Notably, the present study, to the best of our knowledge, is first to demonstrated that UNBS5162, as a naphthalimide, inhibited esophageal cancer cell proliferation, invasion and migration. The effects of UNBS5162 in the present study are all based on results in the KYSE-30 esophageal cancer cell line, and the effects on additional esophageal cancer cell lines should be investigated in future experiments.

The present study also analyzed cell apoptosis and the associated mechanisms in esophageal cancer cells. UNBS5162 was demonstrated to induce cell apoptosis and inhibit the PI3K/AKT signaling pathway. Similarly, a previous study reported that CCR5 blockade was associated with a significant reduction in the migration of microglia, an effect that was mediated via the inhibition of the AKT pathway (30). The present study is the first to illustrate that inhibition of esophageal cancer cells using a CXCL antagonist may occur via inhibition of the PI3K/AKT signaling pathway. Previous studies have indicated that cell proliferation is strongly associated with cell apoptosis in the development of numerous types of cancer (31-35). In the present study, a reduction in cell proliferation was accompanied by increased apoptosis following treatment with UNBS5162, as determined by CCK-8 assays, flow cytometry and alterations in the expression of apoptosis-associated proteins. Caspase-3 is a marker for the induction of apoptosis (36). In the present study, UNBS5162 was demonstrated to activate cell apoptosis. As a result, levels of the antiapoptotic protein $\mathrm{Bcl}-2$ were significantly downregulated, while levels of the proapoptotic protein Bax were increased, which led to caspase-3 upregulation. The results of western blotting indicated that cell apoptosis was stimulated by UNBS5162 treatment, which was consistent with a previous study (29), further confirming that UNBS5162 may exert its effects on tumor growth by apoptotic induction via the Bcl-2-regulated caspase-3 signaling pathway.

Furthermore, the results of the present study indicated that UNBS5162 significantly inhibited the PI3K/AKT signaling pathway in KYSE-30 esophageal cancer cells, as levels of p-AKT, p-mTOR, p70 ${ }^{\mathrm{S} 6 \mathrm{~K}}$ and cyclin D1 were decreased, while increased PDCD4 expression was observed. It is established that the PI3K/AKT pathway regulates various cellular processes, including cell proliferation, apoptosis and tumorigenesis (37). The activation of AKT triggers cyclin D1 expression, which is implicated in the cell proliferation of various cancer cell types, including glioma cells (38). Therefore, inhibiting AKT activation and reducing cyclin D1 expression may suppress cancer cell proliferation. In addition, mTOR was reported to promote the proliferation of cancer cells via $\mathrm{p} 70^{\mathrm{S} 6 \mathrm{~K}}$ stimulation (39). PI3K/AKT affect cell apoptosis through various mechanisms, including regulating the activity of Bcl-2 family members. Bcl-2 family members are divided into antiapoptotic (such as Bcl-2) and proapoptotic (such as Bax) proteins. PI3K-dependent activation of AKT promotes Bcl-2 expression and enhances antiapoptotic effects. At the same time, activation of the PI3K/AKT inactivates Bax, which further contributes to the inhibition of apoptosis (40). Furthermore, PI3K/AKT signaling also affects cell apoptosis by inhibiting caspase-induced cell apoptosis (41), and inhibition of glycogen synthase kinase 3 activity was reported to accelerate apoptosis (42). In addition, activation of AKT induced cell apoptosis through forkhead box O1-Fas ligand signaling (43), and inhibited the release of mitochondrial cytochrome $\mathrm{c}$ and proapoptosis factors, thereby inhibiting apoptosis (44). Consistent with the results of previous studies, the present study demonstrated that inhibition of AKT activity, accompanied by reduced Bcl-2 and enhanced Bax and caspase-3 levels, was associated with increased apoptosis in KYSE-30 esophageal squamous cell carcinoma cells. These results indicate that UNBS5162 may inhibit cell proliferation by suppressing the PI3K/AKT signaling pathway to induce apoptosis.

In conclusion, the present study characterized the anticancer properties and underlying mechanisms of UNBS5162. The results demonstrated that UNBS5162 is a promising anticancer agent for esophageal cancer and may be useful as an adjuvant. The anticancer effect of UNBS5162 via its naphthalimide properties has been illustrated, however, the potential candidate gene which has not been screened out requires substantial amounts of esophageal cancer samples, and further investigation regarding its interactome and the physiological cellular outcomes should be analyzed in future studies.

\section{Acknowledgements}

The present study was supported by Xinjiang Affiliated Tumor Hospital, Xinjiang Medical University Foundation (grant no. 2013-04).

\section{References}

1. Ganeshan D, Bhosale P, Yang T and Kundra V: Imaging features of carcinoid tumors of the gastrointestinal tract. AJR Am J Roentgenol 201: 773-786, 2013.

2. Hunt RH and Yaghoobi M: The esophageal and gastric microbiome in health and disease. Gastroenterol Clin North Am 46: 121, 2017.

3. Awut I, Niyaz M, Biekemitoufu H, Zhang Z, Sheyhedin I and Hao W: Molecular pathological diagnosis for early esophageal cancer in Kazakh patients. Oncol Lett 3: 549-553, 2012.

4. Dong X, Wang L, Han Z, Zhou L, Shan L, Ding Y, Xu W, Li J, Su Y, Cai R, et al: Different functions of DEPTOR in modulating sensitivity to chemotherapy for esophageal squamous cell carcinoma. Exp Cell Res 353: 35-45, 2017.

5. Morimoto A, Oh Y, Nakamura S, Shioda Y, Hayase T, Imamura T, Kudo K and Imashuku S; Japan Langerhans cell histiocytosis Study Group: Inflammatory serum cytokines and chemokines increase associated with the disease extent in pediatric Langerhans cell histiocytosis. Cytokine 97: 73-79, 2017.

6. Otsubo Y, Hashimoto K, Kanbe T, Sumi M and Moriuchi H: Association of cord blood chemokines and other biomarkers with neonatal complications following intrauterine inflammation. PLoS One 12: e0175082, 2017.

7. Zlotnik A and Yoshie O: Chemokines: A new classification system and their role in immunity. Immunity 12: 121-127, 2000.

8. Burkhardt AM, Tai KP, Flores-Guiterrez JP, Vilches-Cisneros N, Kamdar K, Barbosa-Quintana O, Valle-Rios R, Hevezi PA, Zuñiga J, Selman M, et al: CXCL17 is a mucosal chemokine elevated in idiopathic pulmonary fibrosis that exhibits broad antimicrobial activity. J Immunol 188: 6399-6406, 2012.

9. Bruyère C, Lonez C, Duray A, Cludts S, Ruysschaert JM, Saussez S, Yeaton P, Kiss R and Mijatovic T: Considering temozolomide as a novel potential treatment for esophageal cancer. Cancer 117: 2004-2016, 2011. 
10. Sasaki K, Natsugoe S, Ishigami S, Matsumoto M, Okumura H, Setoyama T, Uchikado Y, Kita Y, Tamotsu K, Hanazono K, et al: Expression of CXCL12 and its receptor CXCR4 in esophageal squamous cell carcinoma. Oncol Rep 21: 65-71, 2009.

11. Wang B, Khachigian LM, Esau L, Birrer MJ, Zhao X, Parker MI and Hendricks DT: A key role for early growth response-1 and nuclear factor-kappaB in mediating and maintaining GRO/CXCR2 proliferative signaling in esophageal cancer. Mol Cancer Res 7: 755-764, 2009

12. Blanchet $X$, Langer $M$, Weber $C$, Koenen RR and von Hundelshausen P: Touch of chemokines. Front Immunol 3 $175,2012$.

13. Nomiyama $H$, Hieshima K, Osada N, Kato-Unoki $Y$, Otsuka-Ono K, Takegawa S, Izawa T, Yoshizawa A, Kikuchi Y, Tanase S, et al: Extensive expansion and diversification of the chemokine gene family in zebrafish: Identification of a novel chemokine subfamily CX. Bmc Genomics 9: 222, 2008.

14. Lacalle RA, Blanco R, Carmona-Rodríguez L, Martín-Leal A, Mira E and Mañes S: Chemokine receptor signaling and the hallmarks of cancer. Int Rev Cell Mol Biol 331: 181-244, 2017.

15. Nagarsheth N, Wicha MS and Zou W: Chemokines in the cancer microenvironment and their relevance in cancer immunotherapy. Nat Rev Immunol 17: 559-572, 2017

16. Salazar N, Castellan M, Shirodkar SS and Lokeshwar BL: Chemokines and chemokine receptors as promoters of prostate cancer growth and progression. Crit Rev Eukaryot Gene Expr 23 77-91, 2013.

17. Ransohoff RM: Chemokines and chemokine receptors: Standing at the crossroads of immunobiology and neurobiology. Immunity 31: 711-721, 2009.

18. Balkwill FR: The chemokine system and cancer. J Pathol 226 : 148-157, 2012

19. Jin JJ, Dai FX, Long ZW, Cai H, Liu XW, Zhou Y, Hong Q, Dong QZ, Wang YN and Huang H: CXCR6 predicts poor prognosis in gastric cancer and promotes tumor metastasis through epithelial-mesenchymal transition. Oncol Rep 37: 3279-3286, 2017.

20. Xiang Z, Zhou ZJ, Xia GK, Zhang XH, Wei ZW, Zhu JT, Yu J, Chen W, He Y, Schwarz RE, et al: A positive crosstalk between CXCR4 and CXCR2 promotes gastric cancer metastasis. Oncogene 36: 5122-5133, 2017

21. Dang H, Wu W, Wang B, Cui C, Niu J, Chen J, Chen Z and Liu Y: CXCL5 plays a promoting role in osteosarcoma cell migration and invasion in autocrine- and paracrine-dependent manners. Oncol Res 25: 177-186, 2017.

22. da Silva JM, Moreira Dos Santos TP, Sobral LM Queiroz-Junior CM, Rachid MA, Proudfoot AEI, Garlet GP, Batista AC, Teixeira MM, Leopoldino AM, et al: Relevance of CCL3/CCR 5 axis in oral carcinogenesis. Oncotarget 8: 51024-51036, 2017.

23. Garg R, Blando JM, Perez CJ, Abba MC, Benavides F and Kazanietz MG: Protein kinase $C$ epsilon cooperates with PTEN loss for prostate tumorigenesis through the CXCL13-CXCR5 Pathway. Cell Rep 19: 375-388, 2017.

24. Tian ZY, Xie SQ, Du YW, Ma YF, Zhao J, Gao WY and Wang CJ: Synthesis, cytotoxicity and apoptosis of naphthalimide polyamine conjugates as antitumor agents. Eur J Med Chem 44: 393-399, 2009

25. Ge C, Chang L, Zhao Y, Chang C, Xu X, He H, Wang Y, Dai F, Xie S and Wang C: Design, synthesis and evaluation of naphthalimide derivatives as potential anticancer agents for hepatocellular carcinoma. Molecules 22: E342, 2017.

26. Seliga R, Pilatova M, Sarissky M, Viglasky V, Walko M and Mojzis J: Novel naphthalimide polyamine derivatives as potential antitumor agents. Mol Biol Rep 40: 4129-4137, 2013.

27. Banerjee S, Veale EB, Phelan CM, Murphy SA, Tocci GM, Gillespie LJ, Frimannsson DO, Kelly JM and Gunnlaugsson T: Recent advances in the development of 1,8-naphthalimide based DNA targeting binders, anticancer and fluorescent cellular imaging agents. Chem Soc Rev 42: 1601-1618, 2013.

28. Kamal A, Bolla NR, Srikanth PS and Srivastava AK: Naphthalimide derivatives with therapeutic characteristics: A patent review. Expert Opin Ther Pat 23: 299-317, 2013.
29. Mijatovic T, Mahieu T, Bruyère C,De Nève N, Dewelle J,Simon G, Dehoux MJ, van der Aar E, Haibe-Kains B, Bontempi G, et al: UNBS5162, a novel naphthalimide that decreases CXCL chemokine expression in experimental prostate cancers. Neoplasia 10: 573-586, 2008.

30. Laudati E, Currò D, Navarra P and Lisi L: Blockade of CCR5 receptor prevents M2 microglia phenotype in a microglia-glioma paradigm. Neurochem Int 108: 100-108, 2017.

31. Moradzadeh M, Hosseini A, Erfanian S and Rezaei H: Epigallocatechin-3-gallate promotes apoptosis in human breast cancer T47D cells through down-regulation of PI3K/AKT and telomerase. Pharmacol Rep 69: 924-928, 2017.

32. Geng Y, Zhou Y, Wu S, Hu Y, Lin K, Wang Y, Zheng Z and Wu W: Sulforaphane induced apoptosis via promotion of mitochondrial fusion and ERK1/2-mediated 26S proteasome degradation of novel pro-survival bim and upregulation of bax in human non-small cell lung cancer cells. J Cancer 8: 2456-2470, 2017.

33. Popolin CP, Reis JPB, Becceneri AB, Graminha AE, Almeida MAP, Corrêa RS, Colina-Vegas LA, Ellena J, Batista AA, Cominetti MR, et al: Cytotoxicity and anti-tumor effects of new ruthenium complexes on triple negative breast cancer cells. PLoS One 12: e0183275, 2017.

34. Canesin G, Evans-Axelsson S, Hellsten R, Krzyzanowska A, Prasad CP, Bjartell A and Andersson T: Treatment with the WNT5A-mimicking peptide Foxy-5 effectively reduces the metastatic spread of WNT5A-low prostate cancer cells in an orthotopic mouse model. PLoS One 12: e0184418, 2017.

35. Wang T, Xuan X, Li M, Gao P, Zheng Y, Zang W and Zhao G: Retraction Note: Astragalus saponins affect proliferation, invasion and apoptosis of gastric cancer BGC-823 cells. Diagn Pathol 12: 67, 2017.

36. Ouyang L, Shi Z, Zhao S, Wang FT, Zhou TT, Liu B and Bao JK: Programmed cell death pathways in cancer: A review of apoptosis, autophagy and programmed necrosis. Cell Prolif 45: 487-498, 2012.

37. Hong SY, Yu FX, Luo Y and Hagen T: Oncogenic activation of the PI3K/Akt pathway promotes cellular glucose uptake by downregulating the expression of thioredoxin-interacting protein. Cell Signal 28: 377-383, 2016.

38. Jiang L, Wang C, Lei F, Zhang L, Zhang X, Liu A, Wu G, Zhu J and Song L: miR-93 promotes cell proliferation in gliomas through activation of PI3K/Akt signaling pathway. Oncotarget 6: 8286-8299, 2015.

39. Qiao M, Chang X, Jing F, Rojanasakul Y and Jiang BH: Role of PI3K and AKT specific isoforms in ovarian cancer cell migration, invasion and proliferation through the p70S6K1 pathway. Cell Signal 18: 2262-2271, 2006.

40. Liu G, Song Y, Cui L, Wen Z and Lu X: Inositol hexaphosphate suppresses growth and induces apoptosis in HT-29 colorectal cancer cells in culture: PI3K/Akt pathway as a potential target. Int J Clin Exp Pathol 8: 1402-1410, 2015.

41. YT L, HC W, YC H, CL C, MY Y and CY C: Capsaicin induces autophagy and apoptosis in human nasopharyngeal carcinoma cells by downregulating the PI3K/AKT/mTOR pathway. Int J Mol Sci 18: E1343, 2017.

42. Kanthou C, Dachs GU, Lefley DV, Steele AJ, Coralli-Foxon C, Harris S, Greco O, Dos Santos SA, Reyes-Aldasoro CC, English WR and Tozer GM: Tumour cells expressing single VEGF isoforms display distinct growth, survival and migration characteristics. PLoS One 9: e104015, 2014.

43. Suhara T, Kim HS, Kirshenbaum LA and Walsh K: Suppression of Akt signaling induces fas ligand expression: Involvement of caspase and jun kinase activation in Akt-mediated fas ligand regulation. Mol Cell Biol 22: 680-691, 2002.

44. Caroppi P, Sinibaldi F, Fiorucci L and Santucci R: Apoptosis and human diseases: Mitochondrion damage and lethal role of released cytochrome $\mathrm{C}$ as proapoptotic protein. Curr Med Chem 16: 4058-4065, 2009. 